\title{
Dynamic coordination failures and the efficiency of the firm
}

\author{
Sanjeev Goyal a , Maarten C.W. Janssen ${ }^{\text {b,* }}$ \\ ${ }^{a}$ Econometric Institute, Erasmus University, P.O. Box 1738, 3000 DR Rotterdam, The Netherlands \\ ' Department of Microeconomics, Erasmus University, P.O. Box 1738, 3000 DR Rotterdam, The \\ Netherlands
}

Received 20 April 1993; revised 21 November 1994

\begin{abstract}
This paper examines the role of coordination devices such as work norms in creating and sustaining inefficient organizational practices in firms, in a dynamic environment. The role of signalling norms and product market competition in alleviating such inefficiencies is also examined. In particular, we show that Cournot competition may increase the inefficiency of organizational practices.
\end{abstract}

JEL classification: C72; D21; D82

Keywords: Coordination games; Signalling; Organizational slack; Incentive schemes

\section{Introduction}

In many organizations activities of different employees (or, at a higher level, departments) have to be geared to one another: a certain number of tasks have to be accomplished and it is often more important to ensure that all these tasks will be performed than to ensure that particular persons perform particular tasks. Of course, one allocation of activities to individual employees is often better than another, but any agreement as to who does what is to be preferred to no agreement

\footnotetext{
* Corresponding author
} 
at all. Such an agreement might be called a work norm or an organization code. As organizations are confronted with an environment that changes, the best agreement might change from period to period. The issue we are interested in here is whether individual employees will have adequate incentives to be responsive to such changes. This question is examined in the context of a simple model of a worker-managed firm.

Workers (departments) in the firm are modelled as engaged in interconnected tasks which may be represented by a coordination game. The game has two equilibria which are Pareto-ranked. We introduce the idea that the firm is characterized by a work norm which helps to solve the coordination problem. The work norm asks workers to perform specific tasks (see, Ménard, 1995) and acts as a kind of convention (as in Lewis, 1969). We do not study the question of how the norm comes into existence. Instead, we concentrate on the role of the work norm over time. We consider a dynamic environment in which due to changes within a firm or in a firm's environment, the stage game equilibrium that was the more efficient one at one point in time might become inefficient. We consider situations in which this change is privately observed by one worker and start the analysis by examining whether the work norm will be replaced if it supports a stage-game inefficient outcome.

The first observation is that once a work norm is in place there is an inherent tendency for it to persist even after it is known to be inefficient. This is due to the fact that a pre-existing convention, albeit inefficient, still constitutes an equilibrium of the stage game and if the uninformed worker is not willing to change his behavior the informed worker has no incentive to go in for costly signalling.

This observation motivates our analysis of signalling norms, i.e., examination of equilibria in which communication of change has an impact on future actions via a change of beliefs of the uninformed workers. Our second observation concerns the welfare properties of such equilibria. It is shown that all equilibria that conform to signalling norms can sustain inefficient outcomes as well. In other words, despite the existence of signalling norms, which imply suitable responses by all workers to signals, the incentive to communicate change in stage-game pay-offs might be inadequate. The intuition for this is simple: the cost of change, which is reflected in the loss of some pay-offs in the period of transition, exceeds the benefits (due to an increase in future pay-offs) for the informed player. If one were to interpret a work norm as a "routine" in the sense of Nelson and Winter (1982), then this result provides a formal defense for their claim that routines are relatively inflexible.

This result leads us to examine of the issue of dynamic efficiency, i.e., a concept of efficiency which incorporates the idea of "switching/communication" costs. Our principal result says that all equilibria which conform to stage-game and signalling norms could sustain dynamically inefficient outcomes if individual interests diverge from common interests of the organization.

This conclusion from the analysis of the single firm motivates an analysis of the 
role of product market competition, a la Cournot, in reducing the dynamic inefficiency of organizations. Somewhat surprisingly, it turns out that such competition may have the effect of actually exacerbating inefficiency. The intuition for this result is that a firm can capture more of the benefits of a change in a monopolistic situation than in a more competitive market.

The issues we address in this paper, most notably the consequences of the presence of work norms and conventions, have been discussed in the literature on firms and organizations informally and the purpose of this paper is to argue that these issues can be formalized in a simple way using the framework of (repeated) coordination games. We would like to emphasize that the present paper does not aim to make a contribution to game theory as such, nor does the model presented claim to be a realistic description of organizations. So, for instance, we abstract from conflicts of interest and the presence of hierarchies in organizations. We believe, however, that the results on inefficiency we obtain are likely to obtain in more complicated models of organizations in which conflicts of interest and hierarchies are also incorporated.

Our analysis is related to several strands in the economics literature. We briefly discuss some of these connections now. The possibility of inefficiencies within organizations was recognized early on in Leibenstein's concept of X-efficiency (see Leibenstein, 1966). In later work, Leibenstein (1988), he also examines the role of coordination conventions in organizations in sustaining suboptimal outcomes. This argument is also related to a critique that has been made on a so-called functionalist explanation of organizations, or more generally of institutions. The critique (see, e.g., Basu et al., 1987 and Bianchi, 1995) argues that even though organizations might be efficient at some point in time, their existence at some moment need not imply that they are efficient at that particular point in time. Our formulation of asymmetric information concerning the true pay-off matrix may be interpreted as one of the potential reasons why such inefficiencies may persist.

The impact of competition on reducing managerial slack has also been studied by others (e.g., Hart, 1983 and Scharfstein, 1989). The model we study, however, identifies the slack not in the potential work-shirking indulged in by imperfectly monitored managers, but in coordination costs. To distinguish the two we use the expression organizational slack as against their terminology of managerial slack. Moreover, they look at competitive product markets, whereas we consider oligopolistic markets. Our paper may thus be seen as complementary to the line of research followed in those papers.

Our paper is also related to issues in optimal mechanism/contract design. In particular, the results on dynamic inefficiency raise the question whether there exists an optimal remuneration scheme for workers which can overcome incentive problems arising out of asymmetric information. We show that such a scheme exists in the context of our model, but we argue that since organizations typically face a whole range of potential changes in their environment, many of which 
might be unknown at the moment the remuneration scheme is decided upon, it would be prohibitively expensive to write out a scheme that covers all possible contingencies. The results on dynamic inefficiency of equilibrium outcomes may then be viewed as implications of incomplete contracting (for a survey on contract theory, see Hart and Holmström, 1987).

The paper is organized as follows. Section 2 describes the basic model and shows how equilibria that conform to stage-game and signalling conventions might sustain stage-game inefficient outcomes. (A more formal presentation of some of this material can be found in Goyal and Janssen, 1992). This section also discusses the issue of dynamic ex-post inefficiency in the context of the basic model. Section 3 studies the role of Cournot competition in reducing organizational slack. Section 4 concludes.

\section{The model}

This section consists of five subsections. In subsection 2.1 we describe the basic model in some detail. Subsections 2.2 and 2.3 present the results on stage-game and dynamic efficiency, respectively, while the last two subsections discuss the robustness of our results to the assumptions we make about the way workers are paid (the remuneration scheme) and the way we model communication costs.

\subsection{A simple model}

Consider a worker-managed firm in which two workers have to decide on their effort levels. Each of them can choose to shirk or to work hard. These options are represented by $x_{i}=1$ or 2 , where $x_{i}$ is the effort level of worker $i{ }^{1}$ The production function is represented by

$$
y= \begin{cases}\alpha & \text { if } \min \left(x_{1}, x_{2}\right)=1 \\ \beta & \text { if } \min \left(x_{1}, x_{2}\right)=2\end{cases}
$$

where $y$ is total production of the firm and $\alpha, \beta>0$. It is further assumed that each of the workers gets a share of total production. This share is denoted by $y_{i}$, where $y_{i}=\left(x_{i} / x_{1}+x_{2}\right) y$. Thus, each worker is paid according to his relative effort level. (In subsection 2.4 we discuss the role of this remuneration scheme in our analysis). Finally, the Von Neumann-Morgenstern utility (pay-off) index of worker $i$ is simply represented by $U_{i}\left(x_{i}, x_{-i}\right)=y_{i}-\gamma x_{i}$, where $\gamma>0$. It is thus assumed that an individual worker's direct utility from a low effort level is

\footnotetext{
${ }^{1}$ One can also interpret " 1 " and " 2 "' as two different activities.
} 
positive. On the other hand, he realizes that shirking might have a negative impact on the output that accrues to them. The fact that individual activities have to be geared to each other is formally described in the production technology. A model of this kind has been proposed by Bryant (1983) to analyze macroeconomic coordination failures and by Vega-Redondo (1993) to study the role of "organizational culture". It has also been used in an experimental setting by van Huyck et al. (1990) and Cooper et al. (1990).

The relation between effort and utility levels is summarized in the table below.

\begin{tabular}{ll|c|c|}
\multicolumn{1}{c}{} & \multicolumn{2}{c}{$x_{2}$} \\
\hline$x_{1}$ & 1 & $\frac{\alpha}{2}-\gamma, \frac{\alpha}{2}-\gamma$ & $\frac{\alpha}{3}-\gamma, \frac{2 \alpha}{3}-2 \gamma$ \\
\hline$\frac{2 \alpha}{3}-2 \gamma, \frac{\alpha}{3}-\gamma$ & $\frac{\beta}{2}-2 \gamma, \frac{\beta}{2}-2 \gamma$ \\
\hline
\end{tabular}

The first number gives the utility index of worker 1 . A coordination game results if $U_{1}(2,1)<U_{1}(1,1)$ and $U_{2}(1,2)<U_{2}(2,2)$, i.e., if $\alpha<6 \gamma$ and $2 \alpha+6 \gamma<3 \beta$. In what follows we will assume that this is the case.

The fact that the environment of the firm may change is represented in the simplest possible way. The above coordination game is played a certain number of times. The parameter $\alpha$ is fixed throughout the game; the parameter $\beta$ may take on two values, $\beta_{1}$ and $\beta_{2}$, where $\beta_{2}>\beta_{1}$. At some point in time the value of $\beta$ may change from $\beta_{1}$ to $\beta_{2}$. The values of $\beta_{1}$ and $\beta_{2}$ are such that before the change $\left(x_{1}, x_{2}\right)=(1,1)$ is the more efficient stage-game equilibrium and after the change $\left(x_{1}, x_{2}\right)=(2,2)$ is more efficient, i.e., $\alpha+2 \gamma>\beta_{1}$ and $\alpha+2 \gamma<\beta_{2}$.

Furthermore, we assume that the change is observed privately by one of the players. We think that this is the most usual case in organizations since, due to specialization on a specific task, each worker has specific knowledge about certain aspects of the organization or about the environment. For example, someone working in the purchasing department knows more about the changes that have occurred in the (quality or the prices of the) intermediary products the firm potentially might buy than someone working in the production department and so on. The problem for the worker and the organization is then how this private information can be transferred to the other workers so that a more efficient way of production will be established. Here we assume that the only way of transferring information about change to the other worker is through signalling by means of actions and we will analyze the conditions under which the informed worker will signal the change in pay-offs. This is a simplifying assumption; similar considerations appear, however, if we consider costly "verbal" communication (see subsection 2.5 below). 
The periods in our model can be divided in three: the periods before the change occurs, the period of the change and the periods after the change. We start our analysis at the moment that an agreement (or work norm) of how to play the initial game has been reached, i.e., we do not analyze the question how the initial agreement has come about. The work norm suggests an action for both workers as long as they have not observed a change in the game or a change in the action of their co-worker.

\subsection{Stage-game efficiency}

In the above framework, there are three potential sources of inefficiencies. A (trivial) first source of inefficiency arises when the initial agreement initially supports an inefficient outcome. More concretely, the agreement is inefficient if it suggests that workers choose $x_{i}=2$ before the change has actually occurred. In what follows we will rule out this type of inefficiency by postulating that the initial agreement in the organization suggests actions that support efficient stage game play, i.e. the initial agreement is to play $x_{i}=1$.

The other two sources of inefficiency have to do with the conditions under which the informed worker is willing to signal the change to the other worker. Given that the initial agreement is to play $(1,1)$, an informed worker can signal the change in the production process by playing $x_{i}=2$. The uninformed worker will see that the informed worker has changed his action either by observing the action of the informed worker directly or by observing the change in utility that accrues to him. The uninformed worker can react in two ways to the change of action of his co-worker. Either he interprets the change as a mistake and continues to work at the low effort level or he interprets the change of action as a signal that a change in the production process has taken place and that coordinating on the high effort level is now more efficient. In the second alternative, he may switch effort levels from the next period onwards. Note that the existence of an initial work norm is a necessary (but not a sufficient) condition for the uninformed worker to be able to interpret the change of action as a signal: without the work norm he would probably not even have noticed that the other worker has taken an unusual action.

The second source of inefficiency is then the possibility that the uninformed worker interprets a change of action of the other worker as a mistake. If he does so, there is no incentive for the informed worker to signal, because the uninformed worker will keep playing $x_{i}=1$ and the best response of the informed worker to this strategy is to play $x_{i}=1$ as well. This argument depends on the fact that $\left(x_{1}, x_{2}\right)=(1,1)$ remains an equilibrium in the new stage game. Note that this holds independently of the value of $\beta_{2}$.

Organizations operating in a dynamic environment often develop gradually some procedures for communicating or signalling change. We incorporate this feature in our model by formulating a so-called signalling norm. The signalling 
norm says that workers believe that their colleague-worker will change actions only if he (the colleague) has good reasons to do so, i.e., he has observed a change to the good in stage-game pay-offs. In particular, the uninformed worker will interpret a change in actions of the colleague-worker as a signal that the pay-offs have changed. In order to illustrate the intuition of signalling norms, we will simplify the exposition even further by concentrating the analysis on two periods: a first period in which the change in pay-off might have occurred and the period immediately after that. For simplicity it is assumed that future pay-offs are not discounted, i.e., the discount factor $\delta$ equals $1 .{ }^{2}$ The extensive form of this two period game is given below.

The extensive form describes the situation in which worker 1 is the informed worker. When he has to decide on his first action he knows whether or not the change in pay-off has occurred. When worker 2 decides on his action he is uninformed about the action of worker 1 and about whether or not the change has happened in that period. After the first period, the utility level is revealed to the workers and they also remember their own last action. When worker 2 has to move in the last period, he can infer which action his co-worker has chosen in the previous period, but he still does not know whether the change in the environment has taken place and he is also unaware of the action chosen by the informed worker in the second period. This explains why the information sets are as depicted in Fig. 1.

We are now in the position to discuss the third source of inefficiency. The work norm suggests the action $x_{1}=1$ in the two periods. It is clear that the informed worker does not change actions if the pay-offs do not change. Hence, the workers will follow the work norm and this will give a total pay-off of $\alpha-2 \gamma$ to the workers. If the pay-offs have changed the informed worker might wish to signal this change by choosing $x_{1}=2$ in the period of change. As the uninformed worker does not have reasons to change actions, he will follow the work norm by playing $x_{2}=1$ in the period the change has occurred. ${ }^{3}$ Thus, a change in action results in an initial loss in pay-off. A change in actions is thus only beneficial if the future gains are large enough. The signalling norm helps the workers to coordinate actions when it is clear that one of them does not follow the initial work norm. The argument is the following. When worker 2 is at information set III he knows that worker 1 has deviated in period 1 from the work norm. The signalling norm then says that worker 2 believes that worker 1 has had good reasons to deviate. Looking at the utility worker 1 can get when information set III is reached, one can see that there is only one action combination which could yield

\footnotetext{
${ }^{2}$ The general argument of the paper holds true if the game is played for more than two periods and fulure pay-offs are discounted.

${ }^{3}$ More formally, in a Bayes-Nash equilibrium that conforms to the work norm and the signalling norm, the uninformed worker does not switch actions in the period of change if the probability of change is small enough.
} 


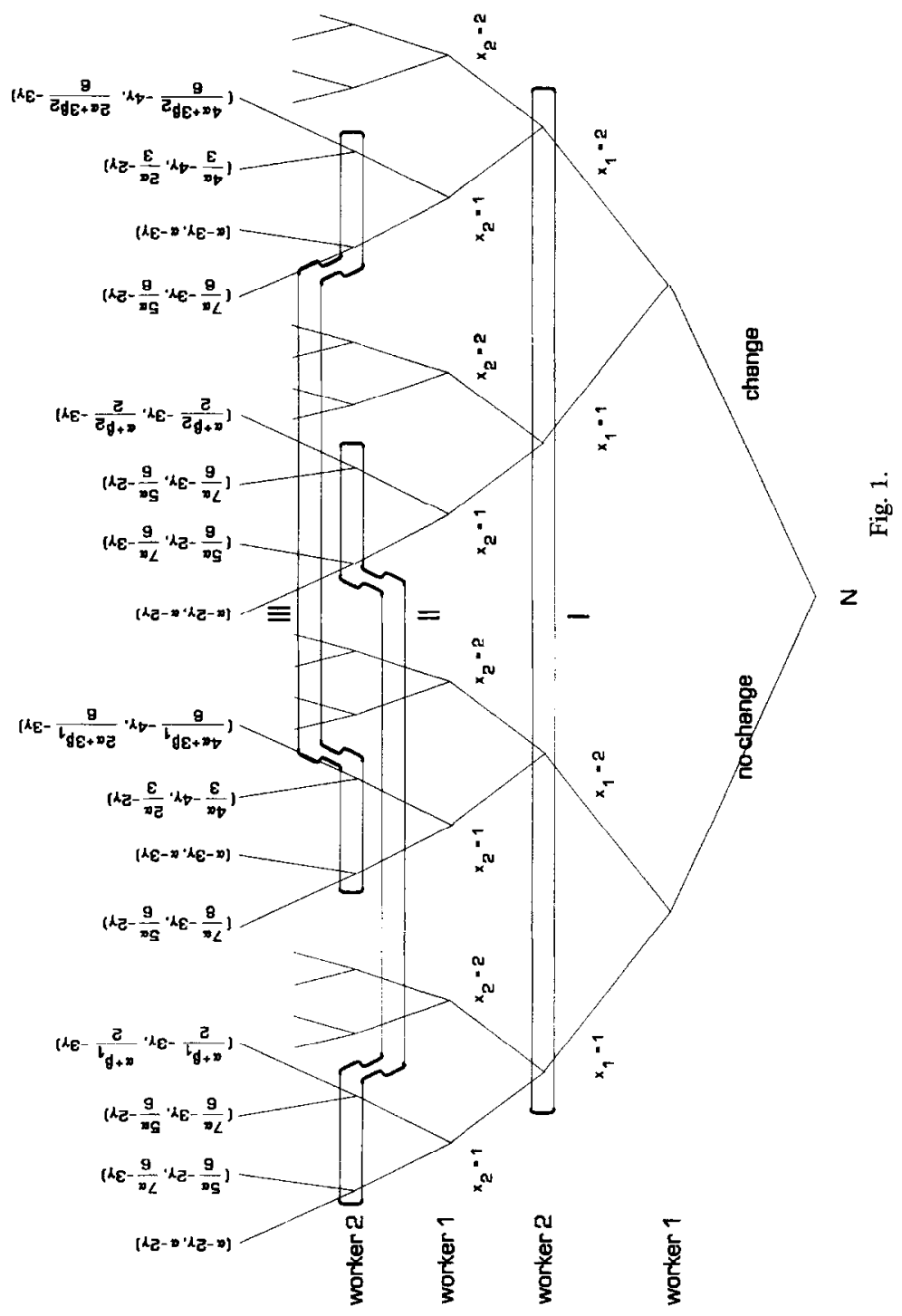


a higher pay-off than $\alpha-2 \gamma$. This action combination is $\left(x_{1}, x_{2}\right)=(2,2)$ and the pay-off to worker 1 is $\left(4 \alpha+3 \beta_{2}\right) / 6-4 \gamma$. The only good reason worker 1 could have had to deviate from the work norm is that $\left(4 \alpha+3 \beta_{2}\right) / 6-4 \gamma>\alpha-2 \gamma$. Worker 2 can then infer that worker 1 will choose $x_{1}=2$ in the second period. Given this inference, worker 2 should also choose $x_{2}=2$ in the second period. Using the signalling norm, worker 1 knows that 2 will play $x_{2}=2$ in the second period when he deviates in the first period. So, he will change actions if $\beta_{2}>2 \alpha / 3+4 \gamma$. The signalling norm will, however, not help the workers to move to the more efficient stage-game cquilibrium in case $\beta_{2}<2 \alpha / 3+4 \gamma$. Thus, in spite of the existence of signalling norms, if $\alpha+2 \gamma<\beta_{2}<2 \alpha / 3+4 \gamma$ players will persist in playing $(1,1)$, which is stage-game inefficient. Thus, the third source of inefficiency is the lack of incentives for the informed worker to signal change. In the next subsection we define dynamic ex-post efficiency and consider the question whether the signalling norm will ensure such efficiency.

The above argument may be summarized in the following result.

\section{Proposition 1}

(a) If $\beta_{2} \in(\alpha+2 \gamma, 2 / 3 \alpha+4 \gamma)$, then a stage-game inefficient mode of behaviour may persist despite the existence of signalling norms.

(b) If $\beta_{2} \geq 2 / 3 \alpha+4 \gamma$, then signalling norms facilitate a switch to stage-game efficient outcomes.

Stage-game inefficiencies might persist in firms in which the workers face a coordination problem because individual workers may have inadequate incentives to communicate (signal) private information. Signalling norms reduce the extent of stage-game inefficiency, but do not eliminate it.

At the end of this subsection it is worth noting that the argument using signalling norms is closely related to what is called "forward induction" in the game theoretic literature (cf., Van Damme, 1989). ${ }^{4}$ By deviating from the work norm it is as if the informed worker sends the following message: "look, we could have got $\alpha-2 \gamma$ if I would not have deviated. I know that you do not know whether there has been a change and which action I will take in the second period. However, the fact that I have deviated and that there is only one action that yields a higher pay-off than we would have obtained otherwise should give you some indication what I will do. So, think well now and decide accordingly". ${ }^{5}$ For this argument to hold true, it is essential that there exists a work norm that initially coordinates the workers" actions. The work norm acts as a kind of "sure outside option".

\footnotetext{
${ }^{4}$ A difference between the concept of forward induction as formulated in Van Damme (1989) and the argument put forward above is that in that paper forward induction is restricted to proper subgames, whereas in the present context the idea of forward induction applies to information sets.

5 This is an adaptation of Van Damme (1989), p. 484.
} 


\subsection{Dynamic ex-post efficiency}

So far we have examined the ex-post stage-game efficiency of equilibria. Such a notion of efficiency is not sensitive to the costs of transition from one norms regime to another. A natural way to extend the idea of stage-game efficiency to incorporate switching or signalling costs is to consider a dynamic notion of efficiency. Since our concern is with inefficiencies at the level of the organization, we will focus on properties of aggregate outcomes. We say that an outcome is dynamically ex-post efficient if there does not exist some other feasible outcome which yields weakly greater aggregate pay-offs in all states of the world and strictly greater pay-offs in some state of the world. The main point of the following analysis is to show that despite the existence of signalling norms, equilibrium outcomes may be dynamically ex-post inefficient.

We need some additional notation: let $S=\left\{\beta_{1}, \beta_{2}\right\}$ be the set of possible states and $U_{i k}(s)$ be the utility of agent $i$ in period $k$ in state $s \in S$. We define the organization's aggregate pay-offs in period $k$ and state $s, U_{k}(s)$, as the sum of the worker's utilities: $U_{k}(s)=U_{1 k}(s)+U_{2 k}(s)$ and the organization's total aggregate pay-offs in state $s, U(s)$, as $U(s)=U_{1}(s)+U_{2}(s)$. Our concern is with dynamic ex-post efficiency and to define this more precisely, we need an understanding of the feasible outcomes. We shall say that a utility outcome $\{U(s)\}_{s \epsilon S}$ is feasible if there exists a strategy combination which can support it. In our context, the key point about strategy combinations is that in period 1 player 2 cannot condition on the true state, and in period 2 he can only condition on the true state if it has been revealed through actions and/or pay-offs in period 1. If we denote by $x_{i k}(s)$ the action of worker $i$ in period $k$ in state $s$, then feasibility requires that $x_{21}\left(\beta_{1}\right)=$ $x_{21}\left(\beta_{2}\right)$ and that $x_{22}\left(\beta_{1}\right)=x_{22}\left(\beta_{2}\right)$ if the true state is not revealed in period 1 .

An outcome $\{U(s)\}_{s \in S}$ is then said to be dynamically ex-post efficient if, and only if, there does not exist another feasible outcome $\left\{U^{\prime}(s)\right\}_{s \epsilon S}$ such that $(i)$ $U^{\prime}(s) \geq \mathrm{U}(s)$ for all $s$ and $(i i) U^{\prime}(s)>U(s)$ for some $s$.

The set of feasible outcomes is rather large, but a moment's reflection reveals that there are only four really interesting strategy combinations when one investigates the efficicncy of a particular outcomc. For casy reference we statc these four combinations here:

(i) $\left\{\forall s, \forall k, \forall i, x_{i k}(s)=1\right\}$

(ii) $\left\{\forall s, x_{11}(s)=x_{21}(s)=1 ; \forall s, x_{12}(s)=x_{22}(s)=2\right\}$

(iii) $\left\{\forall s, x_{11}(s)=x_{21}(s)=2 ; x_{12}(s)=x_{22}(s)=2\right.$ if, and only if, $\left.s=\beta_{2}\right\}$

(iv) $\left\{x_{11}(s)=2\right.$ if, and only if $s=\beta_{2} ; \forall s, x_{21}(s)=1 ; x_{12}(s)=x_{22}(s)=2$ if, and only if, $\left.s=\beta_{2}\right\}$

The first strategy combination refers to the status quo; the second refers to an unconditional change of actions in the second period; the third combination allows the players to condition their strategies on the true state, because they choose 
action combination $(2,2)$ in period 1 ; the fourth combination, finally, incorporates the notion of signalling norms.

Straightforward comparisons of utility levels under these four cases enables us to characterize fully the nature of outcomes that signalling norms can sustain. For expositional clarity, we present the cases of $\alpha>3 \gamma$ and $\alpha<3 \gamma$ separately in the two propositions below.

Proposition 2. Suppose $\alpha<3 \gamma$. Then

(a) if $\beta_{2} \leq \alpha+3 \gamma$, then signalling norms sustain combination (i), which is dynamically efficient.

(b) if $\beta_{2} \epsilon(\alpha+3 \gamma, 2 / 3 \alpha+4 \gamma]$, then signalling norms sustain combination (i), which is dynamically inefficient.

(c) if $\beta_{2}>2 / 3 \alpha+4 \gamma$, then signalling norms sustain combination (iv), which is dynamically efficient.

Proposition 3. Suppose $\alpha>3 \gamma$. Then

(a) if $\beta_{2} \leq 2 / 3 \alpha+4 \gamma$, then signalling norms sustain combination (i), which is dynamically efficient.

(b) if $\beta_{2} \epsilon(2 / 3 \alpha+4 \gamma, \alpha+3 \gamma]$, then signalling norms sustain combination (iv), which is dynamically inefficient.

(c) if $\beta_{2}>\alpha+3 \gamma$, then signalling norms sustain combination (iv), which is dynamically efficient.

These results follow quite directly from the following observations. Using signalling norms, switching from action combination $(1,1)$ to $(2,2)$ involves a signal by the informed player which costs an extra unit of $\gamma$ in period 1 . Recall from subsection 2.2 that action combination $(2,2)$ is stage game superior to $(1,1)$ if, and only if, $\beta_{2}>\alpha+2 \gamma$. This implies that switching is dynamically efficient if, and only if, $\beta_{2}>\alpha+3 \gamma$. Parts $(b)$ of propositions 2 and 3 then follow from the fact that the informed player will signal if, and only if, $\beta_{2}>2 / 3 \alpha+4 \gamma$. Thus, signalling norms can sustain excessive change (Proposition $3 b$ ) as well as tou little change relative (Proposition $2 b$ ) to what is organizationally optimal.

\subsection{The role of specific remuneration schemes}

In our analysis, we have considered a specific remuneration scheme in which workers are paid according to their effort level. Given our results, a natural question that arises is: what is the optimal remuneration scheme and does this optimal scheme sustain dynamic inefficiency? Moreover, if the answer to the last question is negative then what is the significance of our earlier analysis? This subsection addresses these issues.

In general, individual $i$ 's remuneration depends on his effort level and the effort level of the other worker: $y_{i}\left(x_{1}, x_{2}\right), i=1,2$. A feasible remuneration 
scheme is a scheme such that $y_{1}\left(x_{1}, x_{2}\right)+y_{2}\left(x_{1}, x_{2}\right) \leq y\left(x_{1}, x_{2}\right)$. It is easily seen that the scheme

$$
y_{i}\left(x_{1}, x_{2}\right)=\frac{1}{2}\left[y\left(x_{1}, x_{2}\right)+y\left(x_{i}-x_{-i}\right)\right] i=1,2,
$$

is feasible. We will next show that given scheme 2.1 and signalling norms the informed worker will initiate a switch from $\left(x_{1}, x_{2}\right)=(1,1)$ to $\left(x_{1}, x_{2}\right)=(2,2)$ if, and only if, $\beta_{2} \geq \alpha+3 \gamma$, i.e., if and only if, such a switch is dynamically efficient.

Recall from above that an informed worker switches (and signals change in $\beta$ ) if, and only if, pay-offs from doing so exceed the pay-offs of remaining with action combination $(1,1)$. Given scheme $(2.1)$ and the presence of signalling norms this means that an informed worker will switch if, and only if, $2(1 / 2 \alpha-\gamma)<$ $(1 / 2 \alpha+1 / 2 \gamma-2 \gamma)+\left(1 / 2 \beta_{2}-2 \gamma\right)$, which is equivalent to $\beta_{2}>\alpha+3 \gamma$. It then follows from the analysis in the previous subsection that remuneration scheme (2.1) guarantees dynamic efficiency. This result is not very surprising: dynamic inefficiency can only arise in cases in which the interest of the organization does not coincide with the interest of the individual workers. Remuneration scheme (2.1) is constructed in such a way that the interest of the organization and the informed worker always coincide.

The existence of remuneration scheme (2.1) seems to question the robustness of Proposition 2.3. In our view, organizations typically face a variety of uncertainties, many of which may not even be foreseen at the moment a remuneration scheme is contracted upon. This suggests that generally contracts that cover all possible contingencies will be very difficult (and prohibitively expensive) to write out and implement. For a given incomplete contract, there will exist contingencies under which the incentives for the informed worker do not coincide with the interests of the organization. Our analysis in subsections $2.1-2.3$ implicitly assumes the existence of such uncovered contingencies and this motivates the simple remuneration scheme that we study.

\subsection{On the cost of communication}

In the analysis so far we have assumed that the workers cannot verbally communicate with each other. When dealing with organizations this is, of course, a crude assumption to make. However, much of the above analysis remains valid when we allow for costly or noisy communication. In firms, but also in other types of organizations (think, for example, of universities) communication takes place through meetings or via mail. Meetings (as we all know) are costly, because they divert the time of employees away from "directly" productive activities. The assumption that communication is costly reflects these losses of productive time. Mail, on the other hand, is less costly, but also less effective (i.e., more noisy). Letters are not carefully read and the sender of a letter cannot be sure that the information he sends is actually received. 
In the labour-managed firm described above the benefits of a meeting are identical to the benefits of the signalling norm: the information of a pay-off change becomes publicly known and the most appropriate combination of actions will be taken. The cost of a meeting may, however, be larger than the cost of the signalling norm. This is because signalling by changing actions still yields a positive output level in the period of change, while the production process may have to be stopped during a meeting. The exact condition for the signalling norm to be more efficient depends, of course, on how many units of production time a mecting costs and how much effort has to be put into it. It is clear, however, that once the costs of a meeting are taken into account similar considerations apply to the decision whether or not to call for a meeting as to whether or not to signal by changing actions. To illustrate this, in the next section, on the role of competitive pressure, we consider a model which explicitly incorporates communication costs.

\section{Competitive pressure on organizations}

This section examines the extent to which competition can eliminate dynamic ex-post inefficiencies in organizations. We will consider the Cournot form of competition. ${ }^{6}$ For expositional simplicity and following up on the argument concerning communication possibilities, we will reformulate the nature of change in the model. We will, however, retain the basic conceptual apparatus concerning norms (conventions) and equilibria that conform to these norms.

Consider a firm in which two workers have to coordinate their activities to produce some output. There are two possible production processes, denoted by 0 and 1 respectively. The marginal cost of production by means of process 0 is $c_{0}$ in all periods. The marginal costs of process 1 may change over time (due to (say) a variable quality of some of the inputs). Initially, marginal costs of process 1 are $c_{1}$ and $c_{1}>c_{0}$, i.e., initially process 1 is less attractive. Later, the marginal cost of process 1 might be reduced to $c_{2}$ and $c_{2}<c_{0}$, which would make process 1 the cheaper production technology. The firm faces a linear demand curve, represented by $q=1-p$, where $q$ is the output level and $p$ is the price. Thus, the optimal (monopoly) output level is denoted by $\left(1-c_{i}\right) / 2$ and maximal profits are equal to $\left(1-c_{i}\right)^{2} / 4$.

As in the previous section, there is a period in time at which marginal costs for process 1 might switch from $c_{1}$ to $c_{2}$ and we consider a two period case with the period in which change might occur and the period immediately following that. We shall assume that before the change the firm coordinates on process 0 . The

\footnotetext{
${ }^{6}$ An alternative way to model competition is in terms of the Bertrand model of price competition. Here, we consider the Cournot model since (for reasons of simplicity) we want to look at a homogeneous good market and within this context the Bertrand model is not very illuminating.
} 
change is observed privately (say, by the supplies department in the firm) and is not common knowledge. To make the fact commonly known requires some communication among workers. This communication is costly and we assume that it costs $c_{F}>0$ to make the change commonly known and to switch to the more efficient process (if the change has actually taken place). Finally, for convenience, we assume that it takes one period of time to complete the change, i.e., if the change is initiated in period $t$ it is completed only by the beginning of period $t+1$. In line with the analysis of the previous section, we also restrict attention to equilibria in which players conform to stage-game norms in the absence of communication.

What are the conditions for switching to process 1 ? In other words, when does the informed worker communicate his private knowledge. To analyze this question, we specify that the informed worker gets half of total profits and has to incur the fraction $\alpha$ of the communication costs. ${ }^{7}$ Furthermore, for simplicity, we assume workers do not discount future pay-offs. If the cost structure of process 1 changes, the individual pay-off from communicating this change is given by

$$
\frac{1}{2}\left[\left(1-c_{0}\right)^{2} / 4+\left(1-c_{2}\right)^{2} / 4\right]-\alpha c_{F} .
$$

Likewise, the pay-off to the informed worker of not communicating is given by

$$
\left(1-c_{0}\right)^{2} / 4 \text {. }
$$

Thus, communication occurs if, and only if,

$$
2 \alpha c_{F} \leq \frac{1}{4}\left[\left(1-c_{2}\right)^{2}-\left(1-c_{0}\right)^{2}\right] .
$$

It is dynamically ex-post efficient, in the sense defined in the previous section, to switch to process 1 , if and only if, $c_{F} \leq 1 / 4\left[\left(1-c_{2}\right)^{2}-\left(1-c_{0}\right)^{2}\right]$. Accordingly, for $\alpha>1 / 2$ there always exist parameter values of $c_{F}, c_{0}$ and $c_{2}$ such that no switching takes place even though it is dynamically efficient to do so. The level of inefficiency is measured by the difference in pay-off the organization would receive from switching and the pay-off it receives from not switching and is equal to $\left(1-c_{2}\right)^{2} / 4-\left(1-c_{0}\right)^{2} / 4-c_{F}$. This dynamic ex-post inefficiency is due to the mismatch between the incentives of the informed worker and the organization.

We now explore the role of competitive pressure in mitigating this dynamic ex-post efficiency. Competitive pressure is modelled via Cournot competition. We introduce a second firm with a cost structure such that the per unit $\operatorname{cost}$ is $c_{2}$. In the analysis we shall assume, implicitly, that the cost structure of the second firm,

\footnotetext{
${ }^{7}$ As long as the interest of the informed worker does not coincide fully with the interest of the organization, other remuneration schemes would yield conclusions similar to the ones we reach below.
} 
embodied in $c_{2}$ does not reveal any information to firm 1's workers about their own cost structure, i.e., the cost structures of the two firms are uncorrelated. ${ }^{8}$

The question we are interested in is: What is the effect of the presence of a second firm on the incentives for change in firm 1 ? To examine this question, we have to look at the pay-offs of the informed worker, given that the firms play a Cournot-Nash equilibrium in the market game. In case of communication this pay-off is easily calculated to be

$$
\frac{1}{2}\left[\left(1+c_{2}-2 c_{0}\right)^{2} / 9+\left(1-c_{2}\right)^{2} / 9\right]-\alpha c_{F} .
$$

Likewise, the pay-off from not communicating is given by

$$
\left(1+c_{2}-2 c_{0}\right)^{2} / 9 \text {. }
$$

Thus, the informed worker would communicate if, and only if,

$$
2 \alpha c_{F} \leq \frac{1}{9}\left[\left(1-c_{2}\right)^{2}-\left(1+c_{2}-2 c_{0}\right)^{2}\right] \text {. }
$$

As before, if $\alpha>1 / 2$, then there exist parameter values of $c_{F}, c_{0}$ and $c_{2}$ such that no switching takes place even if it is dynamically efficient to do so. In the competition case the level of inefficiency is equal to $\left(1-c_{2}\right)^{2} / 9-\left(1+c_{2}\right.$ $\left.-2 c_{0}\right)^{2} / 9-c_{F}$.

The competition and the monopoly case can be compared by looking at the conditions for switching to the more efficient technology. Thus, we need to look at the following difference of terms:

$$
\left[\left(1-c_{2}\right)^{2} / 4-\left(1-c_{0}\right)^{2} / 4\right]-\left[\left(1-c_{2}\right)^{2} / 9-\left(1+c_{2}-2 c_{0}\right)^{2} / 9\right] \text {. }
$$

Expression (3.7) can be simplified to

$$
\begin{aligned}
& \frac{1}{4}\left(2-c_{0}-c_{2}\right)\left(c_{0}-c_{2}\right)-4 / 9\left(1-c_{0}\right)\left(c_{0}-c_{2}\right) \\
& \quad=\left(c_{0}-c_{2}\right)\left(\frac{1}{18}-\frac{1}{4} c_{2}+\frac{7}{36} c_{0}\right) .
\end{aligned}
$$

Since $c_{2}<c_{0}<1$, both terms are positive. Applying this result to a comparison of Eqs. (3.3) and (3.6) reveals that if the informed worker would switch to the more

\footnotetext{
${ }^{8}$ If the cost structures of the two firms are strongly correlated, the workers in the firm under consideration will be able to infer from the profits they receive in period 1 that technology 1 has become more efficient. This will have the effect that the workers can wait until the second period and if they observe a change in period 1 profits (from what they have "previously" been earning), then both the informed and uninformed worker can switch actions in period 2 without incurring any communication costs. These observations, along with the analysis that follows, suggest that incentives for initiating change for the informed worker will be higher in the case where costs are uncorrelated.
} 
efficient technology in the Cournot competition case, he would also switch in the monopolistic case, but not vice versa. In other words, there are values of $c_{F}$ such that switching to the more efficient technology takes only place in the monopoly case. Accordingly, the "new" technology should be more efficient ( $c_{2}$ should be lower) to make switching beneficial in the competition case than in the monopoly case. Thus, somewhat surprisingly, Cournot competition may actually increase the scope for inefficiency in firms.

The intuition behind this result is that in the case of a monopoly, a firm can capture more of the benefits of a change than in the case of competition. This result is related to the well-known Schumpeterian argument in the literature on innovation that a monopolist might have greater incentives to innovate than a firm in a competitive market (cf., Arrow, 1962).

\section{Conclusion}

This paper has examined the role of coordination devices such as work norms in creating and sustaining inefficient work practices in firms, in a dynamic environment. For certain parameter values, our analysis suggests that once such work norms are in place, there may be an inherent tendency for them to persist, since the individual costs involved in instituting change may be too high relative to the individual benefits. For other parameter values, there may be excess momentum due to the fact that the individual incentives for change do not coincide with the interests of the organization. Furthermore, it is shown that product market competition a la Cournot may aggravate the inefficiency of firms.

\section{Acknowledgements}

This paper is based on (and subsumes) an earlier paper entitled "On The Persistence of Inefficient Norms"'. We have benefitted greatly from presentations at the University of Amsterdam, the Fourgeaud Seminar in Paris, the University of Groningen, The Rationality Session at the World Congress of the International Economic Association, Moscow, the conference "The Emergence and Stability of Institutions", Louvain-la-Neuve and CentER, Tilburg. We would like to thank the participants to the seminars and in particular Dilip Mookherjee and Karl Wärneryd for helpful comments and suggestions. We are also very grateful to the referees for their detailed comments which have led to substantive improvements in the paper.

\section{References}

Arrow, K.J., 1962, Economic welfare and the allocation of resources for inventions, In R. Nelson (ed.), The rate and direction of inventive activity, Princeton, Princeton University Press. 
Basu, K.E., Jones and E. Schlicht, 1987, The growth and decay of custom: The role of the new institutional economics in economic history, Explorations in Economic History, 24, 1-21.

Bianchi, M., 1995, Markets and firms: Transaction costs versus strategic innovation, Journal of Economic Behavior and Organization (this issue).

Bryant, J., 1983, A simple rational expectations Keynes-type model, Quarterly Journal of Economics, 98, 525-9.

Cooper, R. et al., 1990, Selection criteria in coordination games, American Economic Review, 80, 318-33.

Goyal, S. and M. Janssen, 1992, On the persistence of inefficient norms, Research Report 9208 , Econometric Institute, Rotterdam.

Hart, O., 1983, The market mechanism as an incentive scheme, Bell Journal of Economics, 14, 366-82.

Hart, O. and Holmström, B., 1987, The theory of contracts, In Bewley, T. (ed.), Advances in economic theory, Cambridge, Cambridge University Press.

Leibenstein, H., 1966, Allocative vs X-efficiency, American Economic Review, 56, 392-415.

Leibenstein, H., 1988, Inside the firm, Harvard University Press.

Ménard, C., 1995, Markets as institutions versus organizations as markets, Journal of Economic Behavior and Organization (this issue).

Nelson, R. and S. Winter., 1982, An evolutionary theory of economic change, Harvard University Press, Cambridge.

Scharfstein, D., 1989, Product-market competition and managerial slack, Rand Journal of Economics, $19,147-55$.

Van Damme, E., 1989, Stable equilibria and forward induction, Journal of Economic Theory, 48, 476-96.

Vega-Redondo, F., 1993, Competition and culture in an evolutionary process of equilibrium selection; a simple example, Games and Economic Behavior (forthcoming). 